\title{
Deep Learning-Based Assessment of Brain Connectivity Related to Obstructive Sleep Apnea and Daytime Sleepiness
}

\author{
Min-Hee Lee (D) \\ Seung Ku Lee $\mathbb{D}^{\prime}$ \\ Robert J Thomas ${ }^{2}$ \\ Jee-Eun Yoon (iD ${ }^{3}$ \\ Chang-Ho Yun (1D) ${ }^{4, *}$ \\ Chol Shin $\mathbb{D}^{1,5, *}$ \\ 'Institute of Human Genomic Study, College \\ of Medicine, Korea University Ansan \\ Hospital, Ansan, Republic of Korea; \\ ${ }^{2}$ Department of Medicine, Division of \\ Pulmonary, Critical Care and Sleep \\ Medicine, Beth Israel Deaconess Medical \\ Center, Boston, MA, USA; ${ }^{3}$ Department of \\ Neurology, Uijeongbu Eulji Medical Center, \\ Uijeongbu, Republic of Korea; ${ }^{4}$ Department \\ of Neurology, Seoul National University \\ Bundang Hospital, Seongnam, Republic of \\ Korea; ${ }^{5}$ Department of Pulmonary Sleep \\ and Critical Care Medicine Disorder Center, \\ College of Medicine, Korea University, \\ Ansan, Republic of Korea \\ *These authors contributed equally to this \\ work
}

Correspondence: Chang-Ho Yun Department of Neurology, Bundang Clinical Neuroscience Institute, Seoul National University Bundang Hospital, 82 Gumi-ro I73beon-gil, Bundang-gu, Seongnam, 13620, Republic of Korea

Tel +82 31 7877469

Fax +82 31 7874059

Email ych333@gmail.com

Chol Shin

Division of Pulmonary, Sleep, and Critical Care Medicine, Department of Internal Medicine, Korea University Ansan Hospital and Institute of Human Genomic Study, Korea University Ansan Hospital, 516, Gojan-I-dong, Danwongu, Ansan, Gyeonggi-do, I5355,

Republic of Korea

Tel +82 3I 4I2 554I

Fax +82 3I 4I25604

Email chol-shin@korea.ac.kr
Purpose: Obstructive sleep apnea (OSA) is associated with altered pairwise connections between brain regions, which might explain cognitive impairment and daytime sleepiness. By adopting a deep learning method, we investigated brain connectivity related to the severity of OSA and daytime sleepiness.

Patients and Methods: A cross-sectional design applied a deep learning model on structural brain networks obtained from 553 subjects (age, $59.2 \pm 7.4$ years; men, 35.6\%). The model performance was evaluated with the Pearson's correlation coefficient $(R)$ and probability of absolute error less than standard deviation $\left(\mathrm{P}_{\mathrm{AE}<\mathrm{SD}}\right)$ between the estimated and the actual scores. In addition, we investigated sex effects on deep learning outputs for OSA and daytime sleepiness and examined the differences in brain connectivity related to daytime sleepiness between OSA and non-OSA groups.

Results: We achieved a meaningful $R$ (up to 0.74) and $\mathrm{P}_{\mathrm{AE}<\mathrm{SD}}$ (up to 0.92) in a test dataset of whole group and subgroups. Motor, frontal and limbic areas, and default mode network were the prominent hubs of important connectivity to predict OSA severity and daytime sleepiness. Sex affected brain connectivity relevant to OSA severity as well as daytime sleepiness. Brain connectivity associated with daytime sleepiness also differed by the presence vs absence of OSA. Conclusion: A deep learning method can assess the association of brain network characteristics with OSA severity and daytime sleepiness and specify the relevant brain connectivity. Keywords: convolutional neural network, obstructive sleep apnea, daytime sleepiness, diffusion tensor imaging, structural brain network

\section{Introduction}

Sufficient sleep of good quality is essential for optimal brain health. Nevertheless, sleep problems such as insufficient sleep, insomnia, and obstructive sleep apnea (OSA) are common. ${ }^{1,2}$ OSA is characterized by repetitive cessations or reductions in airflow due to upper airway collapse during sleep, resulting in intermittent hypoxia and sleep fragmentation. ${ }^{1}$ OSA not only causes harmful systemic responses including oxidative stress, inflammation, hypertension, and insulin resistance, but also leads to functional impairment and structural alteration of brain. ${ }^{3}$ OSA is related to diminished excessive daytime sleepiness by several mechanisms, including blood-brain barrier dysfunction, ${ }^{4}$ brain edema, ${ }^{5}$ amyloid deposition, ${ }^{6}$ cerebral structure changes, ${ }^{7}$ and altered anatomical brain connections. ${ }^{8,9}$

Discrete brain areas communicate with each other for diverse functions through anatomical connections to generate and integrate information from multiple 
sources. ${ }^{10}$ Structural network properties depend on the integrity of brain structure and connections as well as the efficiency of functional segregation and integration. Altered network properties and their topography can explain neurocognitive defects and symptom diversity in OSA. ${ }^{8,9}$ A good example of phenotypic diversity is excessive daytime sleepiness that does not only impair daytime function and quality of life but also determines cardiometabolic outcomes and therapeutic responses. ${ }^{11,12}$ For the same degree of OSA, the level of daytime sleepiness varies substantially. Its mechanisms are still elusive.

Previous neuroimaging studies highlight the need to address OSA and daytime sleepiness as a network disorder underpinned by brain structure or activity that connects heterogeneous regional function. One study analyzed structural brain networks constructed from diffusion tensor imaging (DTI) and found altered anatomical connections driven by white matter injury in OSA. ${ }^{8}$ Another study demonstrated that OSA led to alterations of global topological characteristics in the brain network constructed by statistical associations of cortical volumes. ${ }^{9}$ Functional MRI studies also report that OSA severity is associated with abnormal functional connectivity as well as cognitive dysfunction. ${ }^{13-16}$ Although very few studies have examined the relationship between daytime sleepiness and brain connectivity, one study reported that daytime sleepiness is associated with functional connectivity in the default mode network (DMN), which has a crucial role in cognition. ${ }^{17}$ An increased rate of $\beta$-amyloid accumulation particularly in the DMN areas is associated with sleepiness. ${ }^{18}$ Residual sleepiness in treated OSA patients may be partially explained by white matter abnormality on DTI. ${ }^{19,20}$ However, it is still unclear which clusters of brain connectivity (ie, pairwise connections between brain regions) specifically correlate with the degree of OSA and daytime sleepiness.

Prior methods for network analysis have limitations in specifying brain connectivity related to OSA severity and daytime sleepiness since it is a complicated task to solve the relationship between a nonlinear complex system (ie, brain network) and continuous measures (ie, degree of OSA severity and daytime sleepiness scale). Thus, it is necessary to develop novel approaches to estimate linear properties from a nonlinear complex system and to understand which brain connections are linearly associated with OSA severity and daytime sleepiness. The deep learning algorithm is a computational technique to assess complex relationships between input features and target variables. ${ }^{21}$
The purpose of this algorithm is to learn a mapping function from input features to targets, achieved by adjusting the weights of the neural network in response to the errors between predicted and actual outputs the model makes on the training dataset. The adjusting processes are intended to continually reduce errors until a good enough model is found. ${ }^{21}$ Contributions of individual input features to performance of decision-making by a trained model can be calculated by gradient information associated with the change in weights in relation to the change in errors (eg, input features with a larger gradient can have a greater effect on the predicted output). ${ }^{22}$ Therefore, deep learning can determine brain connections that are the most susceptible to OSA severity or sleepiness, by maximizing the relationship between predicted and actual scores based on structural network organization while minimizing the errors between the two scores.

The purpose of this study was to investigate anatomical brain connectivity related to OSA severity and daytime sleepiness in a representative sample from a general population in middle-to-late adulthood by using structural network analysis implemented with a deep learning model referred to as BrainNetCNN, which has been recently described as a powerful prediction model for structural brain networks. $^{23}$ We also investigated differences in deep learning outputs according to sex and presence of OSA. Our specific hypothesis was that a deep learning analysis can be predictive of OSA severity and daytime sleepiness. Such an analysis could be an effective tool to improve our understanding of the neuroanatomical substrates for OSA and daytime sleepiness in adult populations.

\section{Methods \\ Study Subjects}

Study procedures were approved by the Institutional Review Board of Korea University Ansan Hospital (approval no. 2006AS0045), which is a sub-study of the Korean Genome and Epidemiology Study (KoGES), an ongoing prospective community-based cohort study. In 2001-2002, the original cohort was established in Ansan, South Korea, and consisted of 5012 adults aged 40-69 years. Furthermore, it was performed in accordance with the principles of the Declaration of Helsinki. Written informed consent from all study participants was obtained. Participants in KoGES have been biennially evaluated for demographic characteristics, medical history, health status, 
and sleep-related factors. As sleep and cognitive aging were adopted as an adjunct research agenda, polysomnography (PSG) and structural MRI were introduced in 2011. In contrast to the core KoGES evaluations performed every 2 years, MRI and PSG was aimed to cover all participants in a 4-year cycle.

For this cross-sectional analysis, we included 625 participants (age, $59.5 \pm 7.6$ years; men, 34.7\%) who completed both MRI and PSG evaluations in 2011. We excluded subjects with 1) major neurological disorders (stroke, $\mathrm{n}=16$; major head trauma, $\mathrm{n}=4$; dementia, $\mathrm{n}=$ 1; hydrocephalus, $\mathrm{n}=4$ ), 2) incomplete Epworth Sleepiness Scale (ESS) assessment $(n=7)$, and 3) loss of MR image slices through the manual review of MRI image quality $(\mathrm{n}=40)$. Finally, 553 participants (age $59.2 \pm 7.4$ years, men $35.6 \%$ ) were included in the present study.

To perform an analysis of brain connectivity related to apnea-hypopnea index (AHI), oxygen desaturation index (ODI), the minimum oxygen saturation $\left(\min \mathrm{SpO}_{2}\right)$ and ESS scores, all subjects were randomly assigned into one of three data-sets: a training set $(n=398)$ to perform deep learning of a connectivity matrix, a validation set $(n=100)$ to confirm convergence of the deep learning model established in the training set, and a test set $(n=55)$ to evaluate the reliability of the deep learning model. In addition, a sex effect on the relationship of brain connectivity with OSA severity and ESS was examined in the subgroup analysis of men ( $n=142 / 35 / 20$ for training/validation/ testing sets $)$ and women $(n=256 / 64 / 36)$. The ESS- associated connectivity was compared between non-OSA ( $\mathrm{n}=222 / 55 / 31$ for training/validation/testing sets) and OSA $(n=176 / 44 / 25)$ groups.

All participants underwent overnight PSG. Apneas and hypopneas were defined according to standard methods. ${ }^{24}$ The AHI was calculated by averaging the total number of obstructive apneas and hypopneas occurring per hour of sleep. OSA was defined as an AHI $\geq 5 /$ hour of sleep and the non-OSA group had an AHI $<5 /$ hour. Daytime sleepiness was assessed using the ESS. ${ }^{25}$ The time interval between overnight PSG and brain MRI was within 2 weeks. The characteristics and sleep variables were presented in Tables 1 and 2. Before the analysis, we investigated the relationship between AHI and ESS to determine whether ESS should be regarded as an independent variable and found no significant correlation $(R=-0.1, P=$ 0.12); therefore, ESS was considered as an independent variable.

\section{Data Acquisition and Processing}

DTI data was acquired using a 1.5-Tesla MRI scanner (General Electric, Milwaukee, WI) at $\mathrm{TR}=15,000 \mathrm{~ms}$, $\mathrm{TE}=93.8 \mathrm{~ms}, 15$ isotropic gradient directions with $\mathrm{b}=$ $1000 \mathrm{~s} / \mathrm{mm}^{2}$, and single $\mathrm{b}=0$ image acquisition. T1weighted images were also obtained with the following parameters: $\mathrm{TR}=7.7 \mathrm{~ms}, \mathrm{TE}=3.4 \mathrm{~ms}$, flip angle $=12^{\circ}$, slice thickness $=1.6 \mathrm{~mm}$. Artifacts including head motion, noise, physiological artifacts, susceptibility-induced distortion, B1 field inhomogeneity, and eddy current-induced

Table I Demographic Data from All Participants

\begin{tabular}{|c|c|c|c|c|c|c|c|}
\hline & Whole $(n=553)$ & Men $(n=197)$ & Women $(n=356)$ & $P$ & Non-OSA $(n=308)$ & OSA $(n=245)$ & $P$ \\
\hline Age (years) & $59.2 \pm 7.4$ & $60.0 \pm 7.3$ & $58.7 \pm 7.5$ & 0.06 & $57.0 \pm 6.4$ & $61.9 \pm 7.7$ & $<0.01$ \\
\hline Men & $197(35.6)$ & & & & $90(29.2)$ & $107(43.7)$ & $<0.01$ \\
\hline BMI $\left(\mathrm{kg} / \mathrm{m}^{2}\right)$ & $24.7 \pm 3.0$ & $24.7 \pm 2.9$ & $24.7 \pm 3.1$ & 0.94 & $23.9 \pm 2.6$ & $25.6 \pm 3.2$ & $<0.01$ \\
\hline \multicolumn{8}{|l|}{ Education } \\
\hline$<6$ years & $101(18.3)$ & $18(9.1)$ & $83(23.3)$ & $<0.01$ & $43(14.0)$ & $58(23.7)$ & $<0.01$ \\
\hline $7-9$ years & $113(20.4)$ & $32(16.2)$ & $81(22.8)$ & 0.07 & $69(22.4)$ & $44(18.0)$ & 0.20 \\
\hline $10-12$ years & $240(43.4)$ & $87(44.2)$ & $153(43.0)$ & 0.79 & $139(45.1)$ & $101(4 \mid .2)$ & 0.36 \\
\hline $13-16$ years & $90(16.3)$ & $53(26.9)$ & $37(10.4)$ & $<0.01$ & $53(17.2)$ & $37(15.1)$ & 0.51 \\
\hline$>16$ years & $9(1.6)$ & $7(3.6)$ & $2(0.5)$ & $<0.01$ & $4(1.3)$ & $5(2.0)$ & 0.49 \\
\hline Current smokers & $47(8.5)$ & $4 \mid(20.8)$ & $6(1.7)$ & $<0.01$ & $24(7.8)$ & $23(9.4)$ & 0.50 \\
\hline Current drinkers & $215(38.9)$ & $122(61.9)$ & $93(26.1)$ & $<0.01$ & $114(37.0)$ & $|0|(4 \mid .2)$ & 0.31 \\
\hline Hypertension & $202(36.5)$ & $83(42.1)$ & $119(33.4)$ & 0.04 & $83(26.9)$ & $119(48.6)$ & $<0.01$ \\
\hline Diabetes mellitus & $132(23.9)$ & $52(26.4)$ & $80(22.5)$ & 0.30 & $46(14.9)$ & $86(35.1)$ & $<0.01$ \\
\hline $\mathrm{BDI}$ & $8.8 \pm 7.4$ & $6.8 \pm 6.7$ & $10.0 \pm 7.5$ & $<0.01$ & $8.8 \pm 6.7$ & $8.9 \pm 8.2$ & 0.88 \\
\hline
\end{tabular}

Notes: Data are presented as $n(\%)$ or mean $\pm S D$, unless otherwise stated.

Abbreviations: OSA, obstructive sleep apnea; BMI, body mass index; BDI, Beck Depression Inventory. 
Table 2 Sleep Variables of All Participants

\begin{tabular}{|c|c|c|c|c|c|c|c|}
\hline & $\begin{array}{l}\text { Whole } \\
(n=553)\end{array}$ & $\begin{array}{c}\text { Men } \\
(n=\mid 97)\end{array}$ & $\begin{array}{l}\text { Women } \\
(n=356)\end{array}$ & $P$ & $\begin{array}{c}\text { Non-OSA } \\
(n=308)\end{array}$ & $\begin{array}{c}\text { OSA } \\
(n=245)\end{array}$ & $P$ \\
\hline $\mathrm{AHI}$ (events/hour) & $6.9 \pm 8.5$ & $8.5 \pm 10.0$ & $6.0 \pm 7.4$ & $<0.01$ & $1.8 \pm 1.4$ & $13.2 \pm 9.5$ & $<0.01$ \\
\hline Mild (5-14) & $175(3 \mid .6)$ & $73(37.1)$ & $102(28.7)$ & 0.04 & & I75 (7I.4) & \\
\hline Moderate (15-29) & $56(10.1)$ & $26(13.2)$ & $30(8.4)$ & 0.07 & & $56(22.9)$ & \\
\hline Severe $(\geq 30)$ & $14(2.5)$ & $8(4.1)$ & $6(1.7)$ & 0.09 & & $14(5.7)$ & \\
\hline${ }_{\min } \mathrm{SpO}_{2}(\%)$ & $87.8 \pm 4.9$ & $86.8 \pm 5.1$ & $88.4 \pm 4.7$ & $<0.01$ & $90.4 \pm 3.1$ & $84.6 \pm 4.8$ & $<0.01$ \\
\hline mean $\mathrm{SpO}_{2}(\%)$ & $95.7 \pm 1.3$ & $95.5 \pm 1.1$ & $95.9 \pm 1.4$ & $<0.01$ & $96.2 \pm 1.2$ & $95.1 \pm 1.2$ & $<0.01$ \\
\hline TS90 (min) & $4.7 \pm 13.3$ & $2.5 \pm 5.5$ & $2.5 \pm 11.8$ & 0.99 & $0.7 \pm 9.5$ & $4.7 \pm 10.2$ & $<0.01$ \\
\hline PTS90 (\%) & $1.5 \pm 3.9$ & $0.7 \pm 1.5$ & $0.7 \pm 3.2$ & 0.99 & $0.2 \pm 2.5$ & $1.3 \pm 2.8$ & $<0.01$ \\
\hline ODI (events/hour) & $6.2 \pm 8.0$ & $7.6 \pm 9.3$ & $5.5 \pm 7.1$ & $<0.01$ & $1.6 \pm 1.4$ & $12.0 \pm 9.0$ & $<0.01$ \\
\hline ESS & $5.0 \pm 3.2$ & $4.6 \pm 2.8$ & $5.3 \pm 3.4$ & 0.01 & $5.2 \pm 3.4$ & $4.9 \pm 3.0$ & 0.03 \\
\hline TST (min) & $391.4 \pm 74.2$ & $384.8 \pm 74.3$ & $395.0 \pm 74.0$ & 0.12 & $395.1 \pm 73.7$ & $386.7 \pm 74.8$ & 0.19 \\
\hline Stage NI (\%) & $6.1 \pm 4.9$ & $6.7 \pm 5.8$ & $5.5 \pm 4.2$ & 0.01 & $5.2 \pm 3.8$ & $6.9 \pm 6.0$ & $<0.01$ \\
\hline Stage N2 (\%) & $56.9 \pm 11.0$ & $56.3 \pm 12.3$ & $57.4 \pm 10.0$ & 0.26 & $57.1 \pm 10.7$ & $56.7 \pm 11.3$ & 0.71 \\
\hline Stage N3 (\%) & $3.2 \pm 4.1$ & $2.4 \pm 4.1$ & $2.6 \pm 3.7$ & 0.51 & $2.7 \pm 3.9$ & $2.3 \pm 3.8$ & 0.23 \\
\hline Stage R (\%) & $20.8 \pm 6.7$ & $20.1 \pm 6.9$ & $21.2 \pm 6.7$ & 0.08 & $21.3 \pm 7.0$ & $20.0 \pm 6.5$ & 0.04 \\
\hline
\end{tabular}

Notes: Data are presented as $n(\%)$ or mean \pm SD, unless otherwise stated.

Abbreviations: $\mathrm{AHI}$, apnea-hypopnea index; ${ }_{\mathrm{min}} \mathrm{SpO}_{2}$, minimum oxygen saturation; ${ }_{\text {mean }} \mathrm{SpO}_{2}$, mean oxygen saturation; ODI, oxygen desaturation index; ESS, Epworth Sleepiness Scale; TST, total sleep time; TS90, time spent with oxygen saturation below $90 \%$; PTS90, percentage of time spent with oxygen saturation below $90 \%$.

distortion in DTI data were corrected by using the FSL eddy tool. ${ }^{26}$ Whole brain streamlines were then reconstructed using second-order integration over fiber orientation distributions ${ }^{27}$ incorporating anatomically constrained tractography ${ }^{28}$ with 1000 dynamically randomized seeding points on the gray-matter/white-matter interface.

A parcellation scheme was applied to whole brain streamlines using an automated anatomical labeling (AAL) atlas, ${ }^{29}$ segmented into 90 cerebral areas in order to construct a structural brain network. Each AAL region represented a node in the brain network, and an edge in the brain network was defined as the number of white-matter streamlines connecting the nodes normalized by volume of the nodes to stabilize inter-subject variability. Before training by using deep learning model, the strength of brain connections was adjusted for age, sex, education, drink, smoke, hypertension, and diabetes mellitus using a multiple linear regression model. For the subgroup analysis by sex, sex was not included in the model. To reduce the effect of inter-subject variability on the training process, the resulting residuals were used to substitute for the raw connection strength.

\section{Conventional DTI Metrics}

To investigate the correlation of conventional DTI metrics including diffusivity and network properties with OSA severity and daytime sleepiness, we employed fractional anisotropy
(FA) and three network parameters including node strength, nodal efficiency, and node betweenness centrality. FA measures the microstructural integrity of white matter. ${ }^{5,8}$ Node strength is the sum of connection weights connecting neighbor nodes, assessing the ability of information interactions with these nodes. ${ }^{30}$ Nodal efficiency reflects the importance of the node for reciprocal communication within the brain network, estimated by the average path length between any node and other nodes. ${ }^{31}$ Node betweenness centrality is defined as the fraction of shortest paths between any two nodes that pass through a given node, and indicates the relative importance of a node on information flow in a complex network. ${ }^{32}$

\section{Convolutional Neural Network}

\section{Architecture}

Before applying the deep learning algorithm to connectivity matrices, as overfitting which corresponds exactly or closely to target variables in the analysis model was expected due to the relatively small size of the training dataset ( $n=398 / 142 / 256 / 222 / 176$ for whole/men/women/ non-OSA/OSA group), we employed the synthetic minority over-sampling technique ${ }^{33}$ based on a randomly interpolated resampling procedure across the nearest neighbors, which enlarges the training dataset by triple augmentations for each subject. 
Prior neural network techniques have a limitation when applied to brain network data to determine the relationship between anatomical brain connectivity related to OSA severity and daytime sleepiness. Fully connected neural networks may ignore the topological relationships between edges or nodes by treating the input connection weights as a vector of features. ${ }^{23}$ Conventional convolutional neural networks (CNNs) use traditional convolutional filters (ie, square filters) designed to capture the spatial $2 \mathrm{D}$ grid locality of images, which treat connectivity matrix as an image. ${ }^{23}$ It also cannot capture topological relationships in the brain network. Therefore, in the present study, we applied the BrainNetCNN, ${ }^{23}$ which is a novel type of CNN optimized for the connectivity matrix. It consists of three types of layers (Figure 1): an Edge-to-Node (E2N) layer, which works by assigning weights to the edges of the network, a node-to-graph (N2G) layer, which reduces the dimensionality of the input by taking a weighted combination of nodes to output a single response and a final fully connected (FC) layer. The E2N layer takes a $90 \times 90$ connectivity matrix derived from DTI and uses a $256 \times 1 \times 90$ filter, which allows to extract topological features in the brain network. It gives a unique output value for each node $i$ as it takes the average of weights of each edge connected to node i. The outputs of the E2N layer, $1 \times 90 \times 256$ feature maps, are the inputs to $\mathrm{N} 2 \mathrm{G}$ layer which acts as a 1D convolution to compress the node information. Finally, the number of features is reduced to the number of target variables through an FC layer with $1 \times 1 \times 64$ feature maps. To avoid overfitting on the training set, we engaged an additional measure, adopting a dropout layer of 0.5 before the FC layer and to prevent vanishing gradient a leaky rectified linear unit with a negative slope of 0.2 was employed. Furthermore, we empirically set the number of training iterations as 30,000 for the whole group and 20,000 for other groups. To minimize the Euclidean distance loss with a weighted L2 regularization term between the predicted and actual scores on the training set, BrainNetCNN was trained using a stochastic gradient

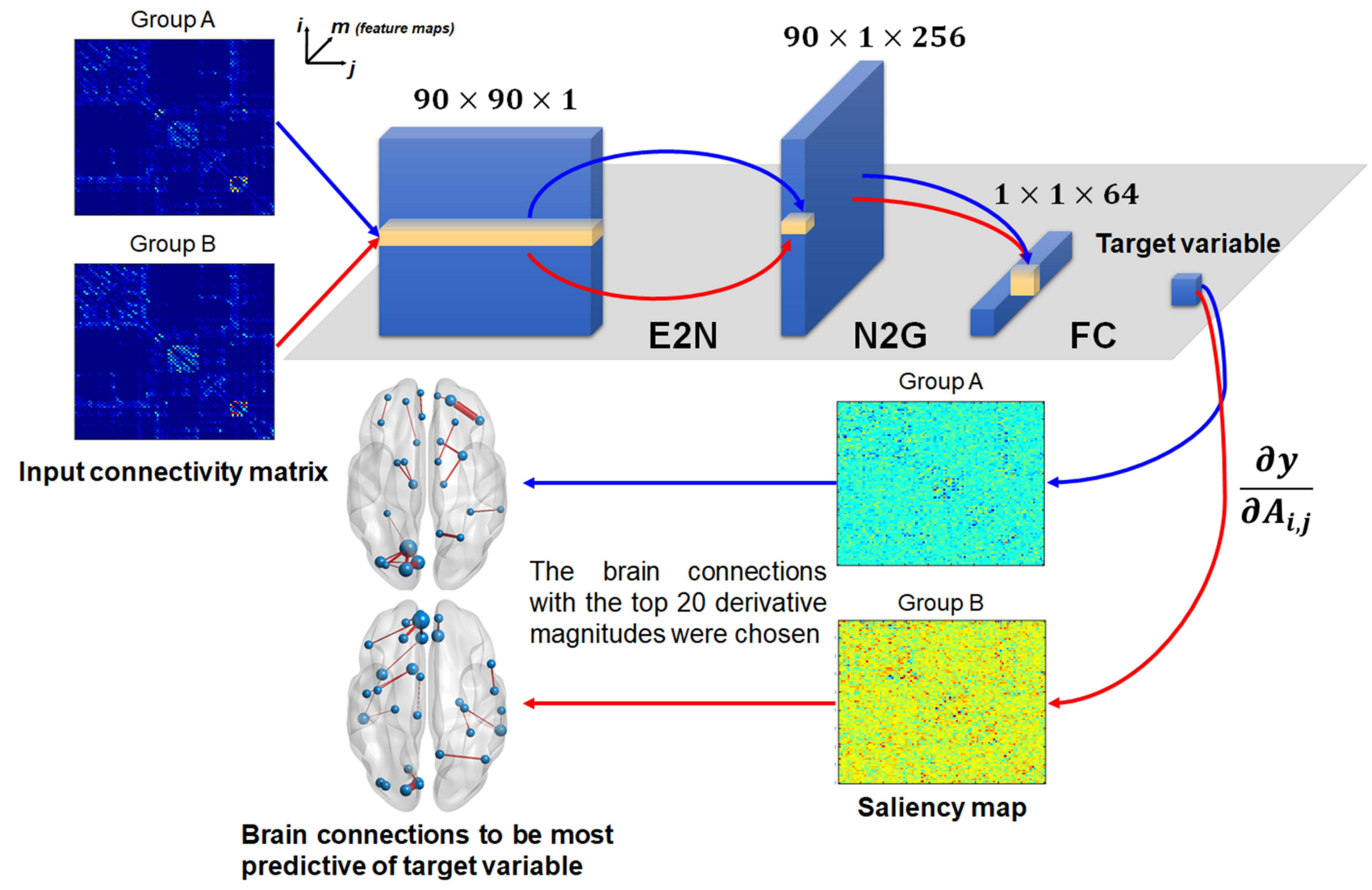

Figure I Schematic overview of the convolutional neural network architecture used to estimate the sleep variables. The convolutional neural network consists of an Edgeto-Node (E2N) layer, a node-to-graph (N2G) layer and fully connected (FC) layer. The E2N layer takes a $90 \times 90$ connectivity matrix and works by assigning weights to the edges of the network, a node-to-graph (N2G) layer reduces the dimensionality of the input by taking a weighted combination of nodes to output a single response and a final fully connected (FC) layer reduces the number of features for estimating a target variable. The saliency map is obtained by computing the partial derivatives of the output with respect to the input connectivity matrix for every input edge. Edges with a larger partial derivative were determined to be more predictive of the target variable. 
descent algorithm, which updates the weights by a linear combination of the negative gradient and the previous weight update at the learning rate of 0.01 .

\section{Statistical Analysis}

The general characteristics are reported as means and standard deviations and proportions with group comparisons using $t$-tests and standard chi-squared test for continuous and categorical variables, respectively. The statistical significance was set at $\mathrm{p}<0.05$. For the conventional DTI metrics analyses, statistical significance was set at $\mathrm{p}<0.05$, after false discovery rate controlled for multiple comparisons.

To evaluate consistency and performance of the BrainNetCNN model, we performed Three-fold CrossValidation on the training and validation sets, and calculated the mean absolute error (MAE), the Pearson's correlation coefficient $(R)$ and the probability of absolute error less than standard deviation $\left(\mathrm{P}_{\mathrm{AE}<\mathrm{SD}}\right)$ between the predicted and the actual scores. In the present study, we report average and standard deviation of MAE, $R$ and $\mathrm{P}_{\mathrm{AE}<\mathrm{SD}}$ over crossvalidation folds. The statistical significance for $R$ was set at $\mathrm{p}<0.05$ in all cross-validations. When the $R$ between the predicted and actual scores met the statistical significance $(p<0.05)$ in all cross-validations, the deep learning model was accepted as a meaningful prediction model. To determine which pairwise connections were learned by BrainNetCNN to be predictive of AHI, ODI, ${ }_{\min } \mathrm{SpO}_{2}$ and ESS, we used the method of saliency visualization ${ }^{22}$ that computes the partial derivatives of the output (ie, predicted score) with respect to the input connectivity matrix for every input edge. ${ }^{23}$ Edges with a larger partial derivative were determined to be more predictive of the actual score, which have higher pairwise connections contributing to better prediction (Figure 1). The BrainNetCNN was implemented with deep a learning framework (Caffe, https:// caffe.berkeleyvision.org/).

\section{Results \\ Conventional DTI Metrics}

There were no meaningful correlations $(R<0.3$ and $P>0.05)$ between any conventional DTI metrics including FA, node strength, regional efficiency, and node betweenness centrality and OSA severity and daytime sleepiness in the whole dataset, and in all the subgroups. This suggests that conventional DTI metrics may not be a proper predictor of OSA severity and daytime sleepiness although they were different by group comparisons between non-OSA and OSA groups as reported in previous studies including our own, based on the same population. $^{8,9}$

\section{Prediction of OSA Severity}

The results of using the BrainNetCNN model to predict AHI, ODI and ${ }_{\min } \mathrm{SpO}_{2}$ scores are summarized in Table 3. We achieved meaningful Pearson's correlation coefficients and $\mathrm{P}_{\mathrm{AE}<\mathrm{SD}}$ for $\mathrm{AHI}$, ODI and ${ }_{\min } \mathrm{SpO}_{2}$ in the test dataset of whole group and the subgroups, suggesting that the deep learning method is better than conventional DTI at predicting the degree of OSA severity indicated by AHI, ODI, and ${ }_{\min } \mathrm{SpO}_{2}$.

Table 3 Pearson's Correlation Coefficient (R), Mean Absolute Error (MAE), and Probability of Absolute Error Less Than Standard Deviation $\left(\mathrm{P}_{\mathrm{AE}<\mathrm{SD}}\right)$ Between Measured and Predicted Apnea-Hypopnea Index (AHI), Oxygen Desaturation Index (ODI), and Minimum Oxygen Saturation $\left({ }_{\min } \mathrm{SpO}_{2}\right)$ Scores with Three-fold Cross-Validation

\begin{tabular}{|c|c|c|c|c|c|c|c|c|c|c|}
\hline & & \multicolumn{3}{|c|}{ AHI } & \multicolumn{3}{|c|}{ ODI } & \multicolumn{3}{|c|}{$\min \mathrm{SpO}_{2}$} \\
\hline & & $R^{*}$ & MAE & $\mathbf{P}_{\mathrm{AE}<\mathrm{SD}}$ & $R^{*}$ & MAE & $\mathbf{P}_{\mathrm{AE}<\mathrm{SD}}$ & $R^{*}$ & MAE & $\mathbf{P}_{\mathrm{AE}<\mathrm{SD}}$ \\
\hline \multirow[t]{3}{*}{ Whole group } & Train & $0.74 \pm 0.0$ & $4.37 \pm 0.3$ & $0.89 \pm 0.0$ & $0.74 \pm 0.0$ & $4.09 \pm 0.3$ & $0.89 \pm 0.0$ & $0.73 \pm 0.0$ & $2.95 \pm 0.3$ & $0.83 \pm 0.0$ \\
\hline & Valid & $0.60 \pm 0.1$ & $5.01 \pm 0.7$ & $0.86 \pm 0.0$ & $0.61 \pm 0.1$ & $4.66 \pm 0.6$ & $0.86 \pm 0.0$ & $0.56 \pm 0.0$ & $3.42 \pm 0.3$ & $0.78 \pm 0.1$ \\
\hline & Test & $0.65 \pm 0.0$ & $6.10 \pm 0.1$ & $0.81 \pm 0.0$ & $0.67 \pm 0.0$ & $5.59 \pm 0.3$ & $0.84 \pm 0.0$ & $0.60 \pm 0.0$ & $3.61 \pm 0.2$ & $0.74 \pm 0.0$ \\
\hline \multirow[t]{3}{*}{ Men group } & Train & $0.95 \pm 0.0$ & $3.60 \pm 0.3$ & $0.95 \pm 0.0$ & $0.95 \pm 0.0$ & $3.13 \pm 0.2$ & $0.94 \pm 0.0$ & $0.92 \pm 0.0$ & $3.10 \pm 0.5$ & $0.83 \pm 0.1$ \\
\hline & Valid & $0.77 \pm 0.1$ & $5.05 \pm 1.2$ & $0.90 \pm 0.1$ & $0.76 \pm 0.1$ & $4.52 \pm 1.1$ & $0.90 \pm 0.1$ & $0.73 \pm 0.1$ & $3.85 \pm 0.1$ & $0.72 \pm 0.0$ \\
\hline & Test & $0.71 \pm 0.0$ & $5.01 \pm 0.3$ & $0.90 \pm 0.1$ & $0.69 \pm 0.0$ & $4.59 \pm 0.2$ & $0.92 \pm 0.0$ & $0.72 \pm 0.0$ & $3.60 \pm 0.5$ & $0.73 \pm 0.0$ \\
\hline \multirow[t]{3}{*}{ Women group } & Train & $0.86 \pm 0.0$ & $3.93 \pm 1.4$ & $0.87 \pm 0.1$ & $0.85 \pm 0.0$ & $3.87 \pm 0.6$ & $0.83 \pm 0.1$ & $0.84 \pm 0.0$ & $2.32 \pm 0.1$ & $0.89 \pm 0.0$ \\
\hline & Valid & $0.77 \pm 0.0$ & $4.47 \pm 0.6$ & $0.84 \pm 0.0$ & $0.75 \pm 0.0$ & $4.32 \pm 0.5$ & $0.82 \pm 0.0$ & $0.60 \pm 0.1$ & $2.86 \pm 0.4$ & $0.86 \pm 0.1$ \\
\hline & Test & $0.73 \pm 0.1$ & $4.22 \pm 1.2$ & $0.85 \pm 0.1$ & $0.74 \pm 0.1$ & $4.21 \pm 0.5$ & $0.82 \pm 0.1$ & $0.55 \pm 0.0$ & $3.04 \pm 0.3$ & $0.78 \pm 0.0$ \\
\hline
\end{tabular}

Notes: *All Pearson's correlation coefficients $(R)$ were statistically significant at $P<0.0$ I. Values are Listed for Three Different Groups/Subgroups: Whole Dataset ( $n=553)$, Men Group $(n=197)$ and Women Group $(n=356)$. 


\section{Anatomical Brain Connectivity Related to OSA Severity}

The partial derivatives predictive of AHI, ODI and min $\mathrm{SpO}_{2}$ scores are plotted with edges connecting nodes of AAL atlas regions (see Figures 2 and 3 ). In the whole group, we could delineate the prominent hubs of important connections such as the left motor area, left fusiform gyrus, and the limbic system, for predicting OSA severity (Figure 2). In the subgroup analysis, anatomical connections related to OSA severity differed by sex (Figure 3). AHI and ODI were predicted by the connections of hubs located in left occipital and right prefrontal areas for men, and in left occipital, prefrontal areas and the limbic system for women. The lowest value of oxygen saturation, $\min \mathrm{SpO}_{2}$, was closely related to left occipital areas and the limbic system in men, and to left occipital, temporal areas and limbic system in women.

\section{Prediction of Degree of Daytime Sleepiness}

The performance of the BrainNetCNN model to predict daytime sleepiness is summarized in Table 4. The deep learning method predicted ESS in the whole group analysis as well as in the subgroup analysis by sex and presence of OSA.
AHI

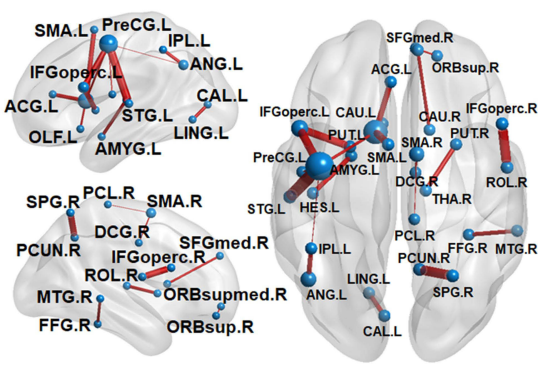

ODI

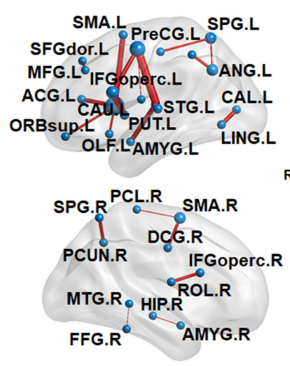

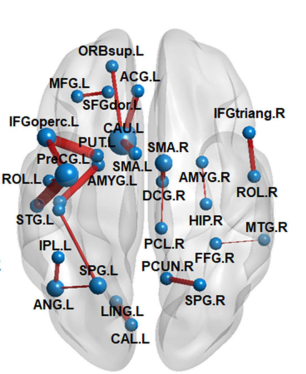

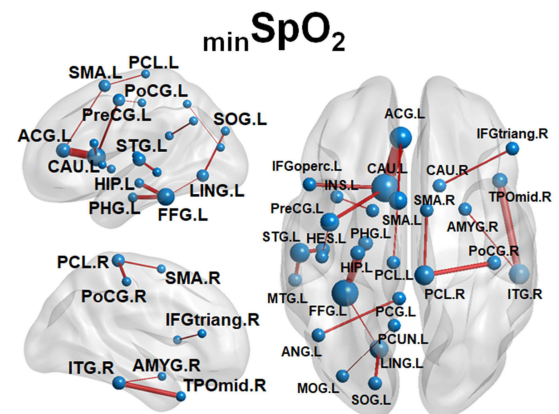

Figure 2 The brain connectivity most predictive of the apnea-hypopnea index (AHI), oxygen desaturation index (ODI), and minimum oxygen saturation (min $\mathrm{SpO}_{2}$ ) scores in the whole group. The brain connectivity with the top 20 derivative magnitudes were chosen for clarity. On the 3D surface images, the thickness of individual edge (red) indicates the partial derivative magnitude and the size of each sphere (blue) indicates the sum of the partial derivative magnitude, with respect to $\mathrm{AHI}, \mathrm{ODI}_{\text {, min } \mathrm{SpO}} \mathrm{S}$ scores, respectively. A complete list of the region labels is available in Table SI.
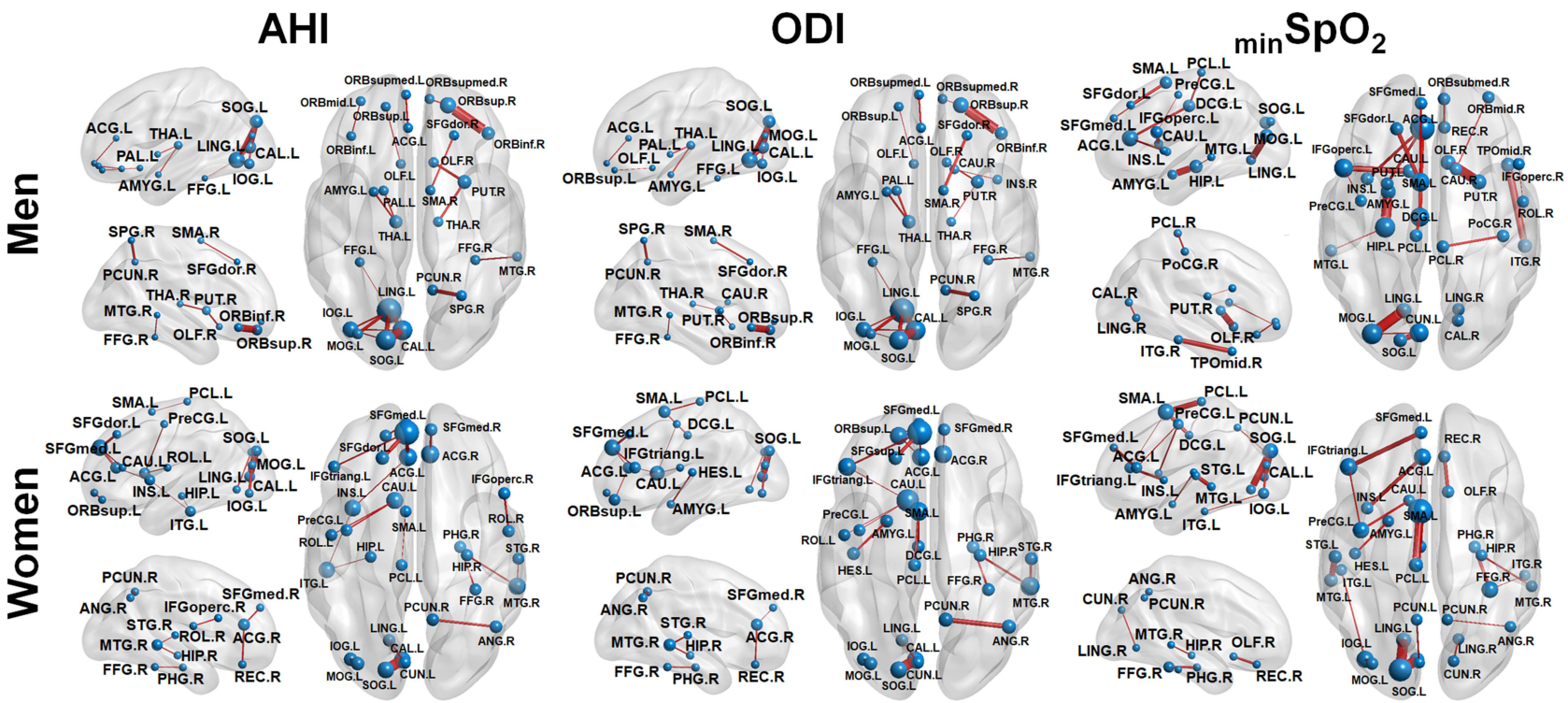

Figure 3 The brain connectivity to be most predictive of apnea-hypopnea index (AHI), oxygen desaturation index (ODI), and minimum oxygen saturation (min $\left.\mathrm{SpO}_{2}\right)$ score for two different subgroups: men and women. The brain connections with the top 20 derivative magnitudes were chosen for clarity. On the $3 D$ surface images, the thickness of individual edge (red) indicates the partial derivative magnitude and the size of each sphere (blue) indicates the sum of the partial derivative magnitude, with respect to AHI, ODI, ${ }_{\min } \mathrm{SpO}_{2}$ scores, respectively. A complete list of region names corresponding to the region labels is available in Table SI. 
Table 4 Pearson's Correlation Coefficient $(R)$, Mean Absolute Error (MAE), and Probability of Absolute Error Less Than Standard Deviation ( $\mathrm{P}_{\mathrm{AE}<\mathrm{SD}}$ ) Between Measured and Predicted Epworth Sleepiness Scale (ESS) Scores with Three-fold CrossValidation

\begin{tabular}{|l|c|c|c|c|}
\hline & & Train Set & Valid Set & Test Set \\
\hline Whole group & $R^{*}$ & $0.75 \pm 0.0$ & $0.60 \pm 0.1$ & $0.54 \pm 0.0$ \\
& $\mathrm{MAE}$ & $1.93 \pm 0.0$ & $2.39 \pm 0.3$ & $2.13 \pm 0.1$ \\
& $\mathrm{P}_{\mathrm{AE}<\mathrm{SD}}$ & $0.82 \pm 0.0$ & $0.72 \pm 0.1$ & $0.76 \pm 0.0$ \\
\hline \multirow{2}{*}{ Men group } & $R^{*}$ & $0.92 \pm 0.0$ & $0.70 \pm 0.0$ & $0.68 \pm 0.1$ \\
& $\mathrm{MAE}$ & $1.73 \pm 0.3$ & $1.94 \pm 0.4$ & $2.39 \pm 0.6$ \\
& $\mathrm{P}_{\mathrm{AE}<\mathrm{SD}}$ & $0.78 \pm 0.1$ & $0.77 \pm 0.1$ & $0.60 \pm 0.1$ \\
\hline \multirow{2}{*}{ Women group } & $R^{*}$ & $0.85 \pm 0.0$ & $0.67 \pm 0.1$ & $0.60 \pm 0.0$ \\
& $\mathrm{MAE}$ & $1.85 \pm 0.2$ & $2.37 \pm 0.3$ & $2.43 \pm 0.2$ \\
& $\mathrm{P}_{\mathrm{AE}<\mathrm{SD}}$ & $0.85 \pm 0.0$ & $0.77 \pm 0.1$ & $0.74 \pm 0.0$ \\
\hline Non-OSA group & $R^{*}$ & $0.87 \pm 0.0$ & $0.74 \pm 0.0$ & $0.73 \pm 0.0$ \\
& $\mathrm{MAE}$ & $1.55 \pm 0.1$ & $2.03 \pm 0.2$ & $2.70 \pm 0.1$ \\
& $\mathrm{P}_{\mathrm{AE}<\mathrm{SD}}$ & $0.92 \pm 0.0$ & $0.81 \pm 0.0$ & $0.72 \pm 0.0$ \\
\hline \multirow{2}{*}{ OSA group } & $R^{*}$ & $0.91 \pm 0.0$ & $0.73 \pm 0.0$ & $0.74 \pm 0.0$ \\
& $\mathrm{MAE}$ & $1.25 \pm 0.1$ & $1.91 \pm 0.1$ & $2.35 \pm 0.1$ \\
& $\mathrm{P}_{\mathrm{AE}<\mathrm{SD}}$ & $0.95 \pm 0.0$ & $0.79 \pm 0.0$ & $0.72 \pm 0.0$ \\
\hline
\end{tabular}

Notes: *All Pearson's correlation coefficients $(R)$ were statistically significant at $P<0.01$. Values are Listed for Five Different Groups/Subgroups: Whole Dataset $(n=553)$, Men Group ( $n=197)$, Women Group $(n=356)$, Non-Obstructive Sleep Apnea (OSA) Group $(n=308)$ and OSA Group $(n=245)$.

\section{Anatomical Brain Connectivity Related to Daytime Sleepiness}

The partial derivatives to be predictive of ESS scores are plotted with edges connecting nodes of the AAL atlas regions (see Figure 4). In the whole group, prominent hubs were the left superior frontal areas, the cingulate, precuneus, angular, occipital temporal, prefrontal, hippocampal, and posterior inferior parietal areas known as components of the right DMN in a previous study. ${ }^{34}$ In the subgroup analysis, sex differences were found in brain connectivity related to daytime sleepiness. The right DMN appeared to be a prominent hub of important connectivity in men. In contrast, the right prefrontal areas and the left DMN were important in women. The presence of OSA also influenced the characteristics of anatomical connections. Important connectivity related to sleepiness were in the right DMN and the left limbic system in the OSA group, but in bilateral prefrontal areas and the right limbic system in the non-OSA group.

\section{Discussion}

This study was motivated by a desire to overcome the limitations of conventional approaches to brain network analyses, which could not readily estimate a continuous property (ie, OSA severity and daytime sleepiness) in relation to a nonlinear complex system (ie, structural brain network). For this goal, we applied a novel deep learning algorithm that can effectively discriminate subtle differences in the strength of anatomical brain connections. Three major findings in the present study support our hypothesis. First, while conventional DTI metrics did not predict OSA severity and daytime sleepiness $(R<0.3$ and $P>0.05)$, the deep learning technique accurately predicted AHI $(R=0.69 \pm 0.1, P<0.01)$, ODI $(R=0.66$ $\pm 0.1, P<0.01),{ }_{\min } \mathrm{SpO}_{2}(R=0.62 \pm 0.1, P<0.01)$, and ESS $(R=0.70 \pm 0.0, P<0.01)$ for test datasets of all group/subgroup analyses. The high correlation as well as the high probability of absolute error within 1 standard deviation (up to 92\%) indicate that the predicted OSA severity and daytime sleepiness is a close proxy of the actual OSA severity and daytime sleepiness. Second, the deep learning technique can determine anatomical brain connectivity associated with OSA and daytime sleepiness. Finally, our approach revealed the effects of sex and OSA on the relationship of anatomical brain connectivity with daytime sleepiness. Sex also influenced network connectivity related to OSA severity. Although previous studies showed group differences, OSA vs non-OSA, in white matter integrity and network properties, ${ }^{5,8,9}$ we could not find any topographic features of FA values and network properties that explained incremental changes in OSA severity and daytime sleepiness with conventional network analysis in this study. The present findings suggest that deep learning techniques are promising tools to provide neuroimaging biomarkers of OSA severity and daytime sleepiness and to explain the phenotypic diversity in OSA.

OSA affects a wide range of brain areas related to medullary respiratory regulatory, cognitive and autonomic function. ${ }^{35}$ In the present study, OSA severity was related to brain connectivity including the left motor system that consists of the precentral gyrus, the supplementary area, and its connections and the left limbic system that includes amygdala, caudate, cingulate, putamen and its neighboring connections. These brain systems are susceptible to OSA and related to breathing control, mood, cognition, and cardiovascular regulation. ${ }^{35}$ These findings suggest that the neural substrates for common clinical manifestations of OSA such as cardiovascular dysregulation, cognitive dysfunction, and affective change can be better identified. Chronic exposure to repetitive hypoxia in OSA results in myelin and axonal damage as well as alterations in the 


\section{Whole}
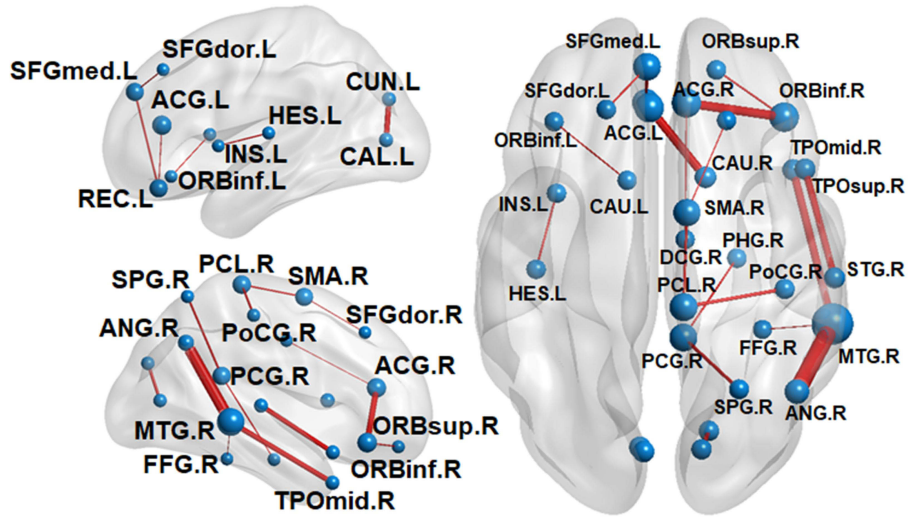

Men

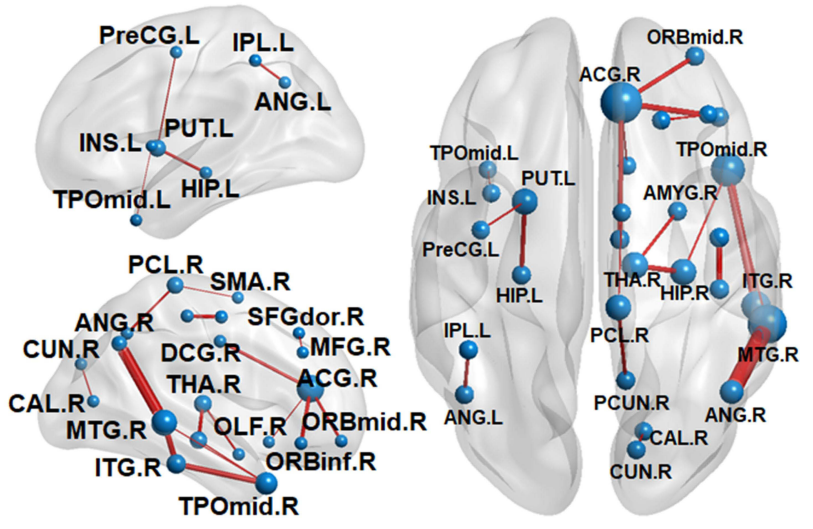

Non-OSA

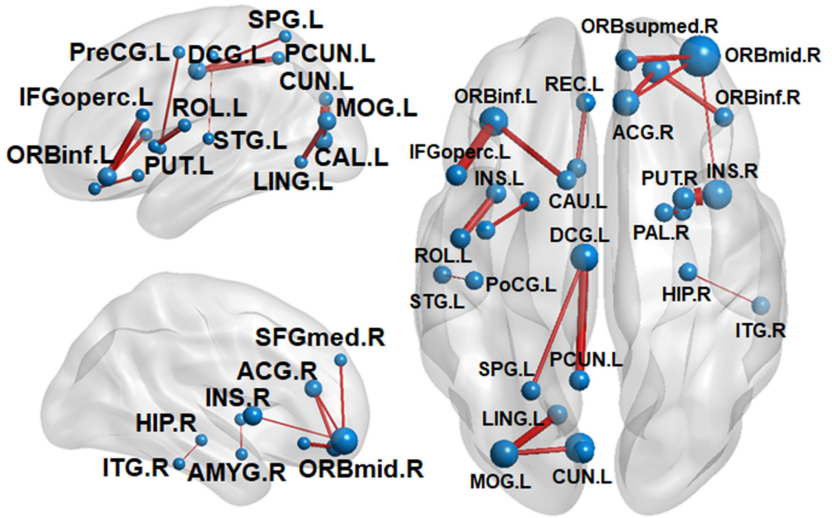

Women

\section{OSA}
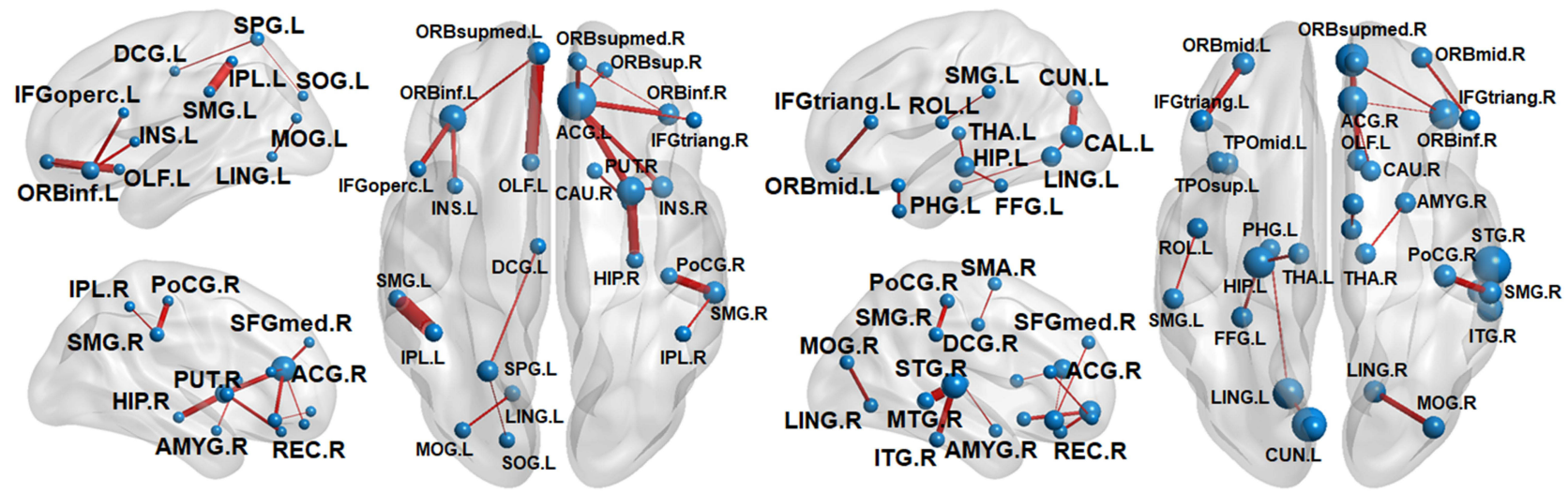

Figure 4 The brain connectivity maps to be most predictive of the Epworth Sleepiness Scale (ESS) scores for five different groups/subgroups: whole dataset, Men group, Women group, non-OSA group and obstructive sleep apnea (OSA) group. The brain connectivity with the top 20 derivative magnitudes were chosen for clarity. On the 3D surface images, the thickness of individual edges (red) indicates the partial magnitude and the size of each sphere (blue) indicates the sum of the partial derivative magnitude, with respect to ESS scores, respectively. A complete list of region names corresponding to the region labels is available in Table SI.

cellular space that lead to changes in structural network properties in these brain areas. ${ }^{8}$ In addition, we found that excessive daytime sleepiness is associated with brain connectivity in the left superior frontal areas and right DMN that consists of cingulate, prefrontal, angular, temporal, and parahippocampus areas. The DMN is closely tied to memory performance ${ }^{36}$ and the prefrontal cortex subserves several cognitive domains. ${ }^{37}$ DMN areas are 
vulnerable to increased amyloid accumulation in older adults who experience daytime sleepiness. ${ }^{18}$ These findings can be interpreted as one of the mechanisms for cognitive impairment in subjects with daytime sleepiness, especially in the elderly population. ${ }^{17}$

The men and women brains show anatomical, functional and biochemical differences across the whole life span. ${ }^{38}$ In the present study, we investigated sex differences in brain connectivity related to OSA severity and daytime sleepiness. Men and women had different patterns of brain connectivity related to AHI, ODI, $\min \mathrm{SpO}_{2}$ and ESS. AHI and ODI was associated with prefrontal area-related connectivity, left side in women but right side in men. The prefrontal area is involved in decision-making and associated with sex differences in its response to stress. ${ }^{39}$ Prefrontal lesions impair decision-making by right-sided lesions in men but by leftsided lesion in women. ${ }^{40}$ In the women, but not in men, AHI and ODI were related to left limbic system-related connectivity. Women have a larger deep limbic system, which has been proposed as one explanation for sex differences in the susceptibility to depression. ${ }^{41}$ Our findings may help explain why depression is reported more frequently as a presenting symptom in women than men with OSA. ${ }^{42}$

The range of excessive daytime sleepiness across severities of sleep apnea or pathological sleep in general remains a mystery. In our analysis, daytime sleepiness was associated with right prefrontal areas and left DMN-related connectivity in women, but with right DMN-related connectivity in men. Daytime sleepiness contributes to memory and mood impairment in OSA. ${ }^{43}$ Interestingly, left and right DMNs are associated with verbal and spatial memory performance, respectively. ${ }^{44}$ In women, right prefrontal areas that play a key role in working memory and executive functioning were also associated with excessive daytime sleepiness, and might be related to the decline in OSArelated attention and executive function. ${ }^{45}$ Sex effects need to be considered when interpreting neuroimaging studies in OSA.

In both groups, anterior cingulate-, parietal-, and orbitofrontal-related connections were common brain connections susceptible to daytime sleepiness. These areas are known to be vulnerable to daytime sleepiness and are associated with increasing amyloid accumulation. ${ }^{18}$ Besides these areas, ESS was related to right limbic system and bilateral prefrontal areas-related connectivity in the non-OSA group, but to right DMN and left limbic system-related connectivity in the OSA group. Affected brain connections were more widely distributed in the
OSA group. In particular, the hippocampus-related connections were susceptible to daytime sleepiness in the OSA group. Hippocampus damage caused by OSA may lead to worsening daytime sleepiness as well as cognitive impairments. ${ }^{46}$

The present study has several limitations. The DTI data had a limited number of isotropic gradient directions $(\mathrm{n}=15)$ and were measured at a relatively low diffusion weight $\left(b=1000 \mathrm{~s} / \mathrm{mm}^{2}\right)$. Thus, the tractography analysis may not be fully resolved in voxels with crossing, kissing, fanning, and curving fiber configurations although we utilized state-of-the-art algorithms. ${ }^{27,28}$ This may lead to incorrect and ambiguous estimates of fiber orientation. ${ }^{47}$ The sample size $(n=553)$ in this retrospective and observational study is not large enough to determine the heterogeneity of high dimensional structural brain network data by using the present deep learning technique. To minimize this limitation, we applied data augmentation. Since this approach may also cause model overfitting for the training dataset, our approach needs to be tested and refined in a larger independent sample including other institutional data sets. It is likely that different severities and clinical vs population-based differences exist, which our results do not address, nor the dynamics of network connectivity over time. Racial differences in connectivity and OSA impact will need assessments in a diverse population. Our subjects were a non-clinical population, mostly diagnosed with milder OSA (71.4\%). Although our trained model successfully predicted OSA severity and found OSA severity-related brain connections, the number of severe OSA was quite small during the training process. Therefore, since there might be the probability of large absolute error for severe OSA, additional validation is needed for severe OSA. In addition, only $6.2 \%$ of our subjects experienced excessive daytime sleepiness (ESS $>10$ ), which may result from underestimation in ESS domains in older adults. ${ }^{48}$ Therefore, although our trained model successfully predicted ESS, the number of severe ESS was relatively small during the training process; additional validation is needed for severe subjective sleepiness. Finally, in the present study, we did not compare cognitive performance between groups. Although most brain connections susceptible to OSA severity and daytime sleepiness determined by deep learning model are related to cognitive performance, decline associated with OSA and sleepiness could not be quantitatively explained. It remains an important line of inquiry for future research. 


\section{Conclusion}

In summary, the present study provides a novel approach to determine the anatomical blueprint underlying OSA and daytime sleepiness-related brain connectivity in middle and late adulthood. This approach has clinical implications to delineate the mechanism of functional impairment and daytime sleepiness, and to predict the risk of subsequent cognitive impairment in OSA.

\section{Acknowledgments}

This research is supported by the grants from the Korea Centers for Disease Control and Prevention (2011-E71004 -00), from the Basic Science Research Program (NRF2019R1A2C2086705), and from the Brain Pool Program (NRF-2020H1D3A1A04105299) through the National Research Foundation of Korea, funded by the Ministry of Science and ICT.

\section{Disclosure}

Dr R. J. Thomas reports personal consulting fees from GLG Councils, Guidepoint Global, and Jazz Pharmaceuticals, outside the submitted work. In addition, RJT has a patent ECG-spectrogram with royalties paid by MyCardio, LLC, a patent PAPGAM issued, and a patent Auto CPAP licensed to DeVilbiss-Drive. All other authors declare no potential conflict of interest.

\section{References}

1. Grandner MA. Sleep, health, and society. Sleep Med Clin. 2017;12:1-22.

2. Veasey SC, Rosen IM. Obstructive sleep apnea in adults. $N$ Engl $J$ Med. 2019;380(15):1442-1449. doi:10.1056/NEJMcp1816152

3. Lévy P, Kohler M, Mcnicholas WT, et al. Obstructive sleep apnoea syndrome. Nat Rev Dis Primers. 2015;1:1-21.

4. Palomares JA, Tummala S, Wang DJ, et al. Water exchange across the blood-brain barrier in obstructive sleep apnea: an MRI diffusion-weighted pseudo-continuous arterial spin labeling study. J Neuroimaging. 2015;25(6):900-905. doi:10.1111/jon.12288

5. Baril AA, Gagnon K, Descoteaux M, et al. Cerebral white matter diffusion properties and free-water with obstructive sleep apnea severity in older adults. Hum Brain Mapp. 2020;41(10):2686-2701. doi:10.1002/hbm.24971

6. Yun CH, Lee HY, Lee SK, et al. Amyloid burden in obstructive sleep apnea. J Alzheimers Dis. 2017;59(1):21-29. doi:10.3233/JAD-161047

7. Baril AA, Gagnon K, Brayet P, et al. Gray matter hypertrophy and thickening with obstructive sleep apnea in middle-aged and older adults. Am J Respir Crit Care Med. 2017;195(11):1509-1518. doi:10.1164/rccm.201606-12710C

8. Lee $\mathrm{MH}$, Yun $\mathrm{CH}$, Min A, et al. Altered structural brain network resulting from white matter injury in obstructive sleep apnea. Sleep. 2019;42(9):zsz120. doi:10.1093/sleep/zsz120

9. Luo Y, Wang D, Liu K, et al. Brain structure network analysis in patients with obstructive sleep apnea. PLoS One. 2015;10(9): e0139055. doi:10.1371/journal.pone.0139055
10. Sporns O, Tononi G, Kötter R. The human connectome: a structural description of the human brain. PLoS Comput Biol. 2005;1(4):e42. doi:10.1371/journal.pcbi.0010042

11. Barnes M, Houston D, Worsnop CJ, et al. A randomized controlled trial of continuous positive airway pressure in mild obstructive sleep apnea. Am J Respir Crit Care Med. 2002;165(6):773-780. doi:10.1164/ajrccm.165.6.2003166

12. Weaver TE, Mancini C, Maislin G, et al. Continuous positive airway pressure treatment of sleepy patients with milder obstructive sleep apnea. Am J Respir Crit Care Med. 2012;186(7):677-683. doi:10.1164/rccm.201202-0200OC

13. Qin Z, Kang D, Feng X, Kong D, Wang F, Bao H. Resting-state functional magnetic resonance imaging of high altitude patients with obstructive sleep apnoea hypopnoea syndrome. Sci Rep. 2020;10 (1):15546. doi:10.1038/s41598-020-72339-2

14. Park B, Palomares JA, Woo MA, et al. Disrupted functional brain network organization in patients with obstructive sleep apnea. Brain Behav. 2016;6(3):e00441. doi:10.1002/brb3.441

15. Chen L, Fan X, Li H, et al. Topological reorganization of the default mode network in severe male obstructive sleep apnea. Front Neurol. 2018;9:363. doi:10.3389/fneur.2018.00363

16. Canessa N, Castronovo V, Cappa SF, et al. Sleep apnea: altered brain connectivity underlying a working-memory challenge. Neuroimage Clin. 2018;19:56-65. doi:10.1016/j.nicl.2018.03.036

17. Ward AM, McLaren DG, Schultz AP, et al. Daytime sleepiness is associated with decreased default mode network connectivity in both young and cognitively intact elderly subjects. Sleep. 2013;36 (11):1609-1615. doi:10.5665/sleep.3108

18. Carvallho D, Louis ES, Knopman D, et al. Excessive daytime sleepiness predicts increased $\beta$-amyloid accumulation in nondemented elderly: a longitudinal PiB-PET study. Neurology. 2017;88:S14.004.

19. Zhang J, Weaver TE, Zhong Z, et al. White matter structural differences in OSA patients experiencing residual daytime sleepiness with high CPAP use: a non-Gaussian diffusion MRI study. Sleep Med. 2019;53:51-59. doi:10.1016/j.sleep.2018.09.011

20. Xiong Y, Zhou XJ, Nisi RA, et al. Brain white matter changes in CPAP-treated obstructive sleep apnea patients with residual sleepiness. J Magn Reson Imaging. 2017;45(5):1371-1378. doi:10.1002/jmri.25463

21. Lecun Y, Bengio Y, Hinton G. Deep learning. Nature. 2015;521 (7553):436-444. doi:10.1038/nature14539

22. Simonyan K, Vedaldi A, Zisserman A. Deep inside convolutional networks: visualising image classification models and saliency maps. arXiv Preprint arXiv. 2013. Available form: https://arxiv.org/pdf/ 1312.6034.pdf. Accessed September 14,2021.

23. Kawahara J, Brown CJ, Miller SP, et al. BrainNetCNN: convolutional neural networks for brain networks; towards predicting neurodevelopment. Neuroimage. 2017;146:1038-1049. doi:10.1016/ j.neuroimage.2016.09.046

24. Iber C, Ancoli-Israel S, Chesson AL, Quan SF. The AASM Manual for the Scoring of Sleep and Associated Events: Rules, Terminology and Technical Specifications. IL: American Academy of Sleep Medicine; 2007.

25. Johns MW. A new method for measuring daytime sleepiness: the Epworth sleepiness scale. Sleep. 1991;14(6):540-545. doi:10.1093/ sleep/14.6.540

26. Andersson JLR, Graham MS, Drobnjak I, et al. Towards a comprehensive framework for movement and distortion correction of diffusion MR images: within volume movement. Neuroimage. 2017;152:450-466. doi:10.1016/j.neuroimage.2017.02.085

27. Tournier JD, Calamante F, Connelly A. Improved probabilistic streamlines tractography by 2 nd order integration over fibre orientation distributions. Proc Int Soc Magn Reson Med. 2010;1670. Available from:https://cds.ismrm.org/protected/10MProceedings/ PDFfiles/1670_4298.pdf. Accessed September 14,2021. 
28. Smith RE, Tournier JD, Calamante F, Connelly A. Anatomicallyconstrained tractography: improved diffusion MRI streamlines tractography through effective use of anatomical information. Neuroimage. 2012;62(3):1924-1938. doi:10.1016/j.neuroimage.20 12.06.005

29. Tzourio-Mazoyer N, Landeau B, Papathanassiou D, et al. Automated anatomical labeling of activations in SPM using a macroscopic anatomical parcellation of the MNI MRI single-subject brain. Neuroimage. 2002;15(1):273-289. doi:10.1006/nimg.2001.0978

30. Rubinov M, Sporns O. Complex network measures of brain connectivity: uses and interpretations. Neuroimage. 2010;52(3):1059-1069. doi:10.1016/j.neuroimage.2009.10.003

31. Achard S, Bullmore E. Efficiency and cost of economical brain functional networks. PLoS Comput Biol. 2007;3(2):e17. doi:10.1371/journal.pcbi.0030017

32. Freeman LC. A set of measures of centrality based on betweenness. Sociometry. 1977;40(1):35-41. doi:10.2307/3033543

33. Chawla NV, Bowyer KW, Hall LO, Kegelmeyer WP. SMOTE: synthetic minority over-sampling technique. $J$ Artif Intell Res. 2002;16:321-357. doi:10.1613/jair.953

34. Mars RB, Neubert FX, Noonan MP, Sallet J, Toni I, Rushworth MFS. On the relationship between the "default mode network" and the "social brain". Front Hum Neurosci. 2012;6:189. doi:10.3389/ fnhum.2012.00189

35. Kumar R, Pham TT, Macey PM, Woo MA, Yan-Go FL, Harper RM. Abnormal myelin and axonal integrity in recently diagnosed patients with obstructive sleep apnea. Sleep. 2014;37(4):723-732. doi: $10.5665 /$ sleep. 3578

36. Anticevic A, Repovs G, Shulman GL, Barch DM. When less is more: TPJ and default network deactivation during encoding predicts working memory performance. Neuroimage. 2010;49(3):2638-2648. doi:10.1016/j.neuroimage.2009.11.008

37. Greicius MD, Krasnow B, Reiss AL, Menon V. Functional connectivity in the resting brain: a network analysis of the default mode hypothesis. Proc Natl Acad Sci USA. 2003;100(1):253-258. doi:10.1073/pnas.0135058100

38. Davies W, Wilkinson LS. It is not all hormones: alternative explanations for sexual differentiation of the brain. Brain Res. 2006;1126 (1):36-45. doi:10.1016/j.brainres.2006.09.105
39. Bland ST, Schmid MJ, Der-avakian A, Watkins LR, Spencer RL, Maier SF. Expression of C-Fos and BDNF mRNA in subregions of the prefrontal cortex of male and female rats after acute uncontrollable stress. Brain Res. 2005;1051(1-2):90-99. doi:10.1016/j. brainres.2005.05.065

40. Tranel D, Damasio H, Denburg NL, Bechara A. Does gender play a role in functional asymmetry of ventromedial prefrontal cortex? Brain. 2005;128(12):2872-2881. doi:10.1093/brain/awh643

41. Zaidi ZF. Gender differences in human brain: a review. Open Anat J. 2010;2:37-55. doi:10.2174/1877609401002010037

42. Yaffe K, Laffan AM, Harrison SL, et al. Sleep-disordered breathing, hypoxia, and risk of mild cognitive impairment and dementia in older women. J Am Med Assoc. 2011;306:613-619.

43. Guilleminault C, Brooks SN. Excessive daytime sleepiness: a challenge for the practising neurologist. Brain. 2001;124 (8):1482-1491. doi:10.1093/brain/124.8.1482

44. Banks SJ, Zhuang X, Bayram E, et al. Default mode network lateralization and memory in healthy aging and alzheimer's disease. J Alzheimers Dis. 2018;66(3):1223-1234. doi:10.3233/JAD-180541

45. Hishikawa N, Fukui Y, Sato K, Ohta Y, Yamashita T, Abe K. Cognitive and affective functions associated with insomnia: a population-based study. Neurol Res. 2017;39(4):331-336. doi: $10.1080 / 01616412.2017 .1281200$

46. Lal C, Weaver TE, Bae CJ, Strohl KP. Excessive daytime sleepiness in obstructive sleep apnea. Mechanisms and clinical management. Ann Am Thorac Soc. 2021;18(5):757-768. doi:10.1513/ AnnalsATS.202006-696FR

47. Thomas C, Ye FQ, Irfanoglu MO, et al. Anatomical accuracy of brain connections derived from diffusion MRI tractography is inherently limited. Proc Natl Acad Sci USA. 2014;111(46):16574-16579. doi:10.1073/pnas.1405672111

48. Onen F, Moreau T, Gooneratne NS, Petit C, Falissard B, Onen SH. Limits of the Epworth sleepiness scale in older adults. Sleep Breath. 2013;17(1):343-350. doi:10.1007/s11325-012-0700-8
Nature and Science of Sleep

\section{Publish your work in this journal}

Nature and Science of Sleep is an international, peer-reviewed, open access journal covering all aspects of sleep science and sleep medicine, including the neurophysiology and functions of sleep, the genetics of sleep, sleep and society, biological rhythms, dreaming, sleep disorders and therapy, and strategies to optimize healthy sleep.
The manuscript management system is completely online and includes a very quick and fair peer-review system, which is all easy to use. Visit http://www.dovepress.com/testimonials.php to read real quotes from published authors. 